Sof t war e- Def i ned Vehi cul ar Net wor ks: Archi t ect ure, Al gor i thms, and Appl i cat i ons: Part 2

\begin{tabular}{|l|l|}
\hline 著者 & $\begin{array}{l}\text { HAN Guangj i e, GUl ZAN Nohsen, BI Yuanguo, LUAN } \\
\text { Tom H. OTA Kaor u, ZHOU Hai bo, GUl BENE Whel, } \\
\text { RAYES Ammar }\end{array}$ \\
\hline $\begin{array}{l}\text { j our nal or } \\
\text { publ i cat i on t i t l e }\end{array}$ & I EEE Communi cat i ons Nagazi ne \\
\hline vol une & 55 \\
\hline number & 8 \\
\hline page r ange & $58-59$ \\
\hline year & $2017-08$ 09 \\
\hline URL & ht t p: //hdl . handl e. net /10258/00009571 \\
\hline
\end{tabular}




\section{SOFTWARE-DEFINED VEHICULAR NETWORKS: ArChiteCture, AlgOrithmS, AND ApplicatiOnS: PART 2}

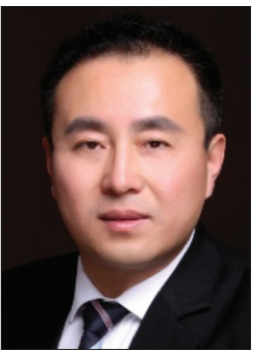

Guangjie Han

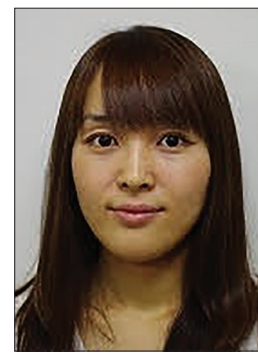

Kaoru Ota

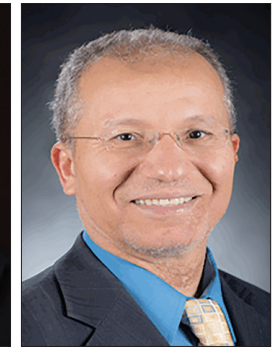

Mohsen Guizani

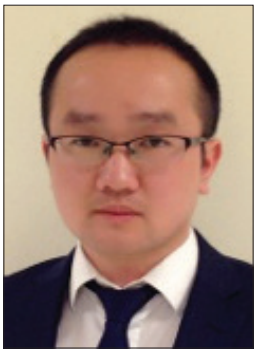

Haibo Zhou

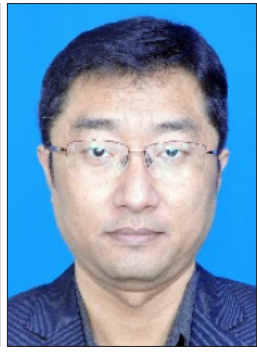

Yuanguo Bi

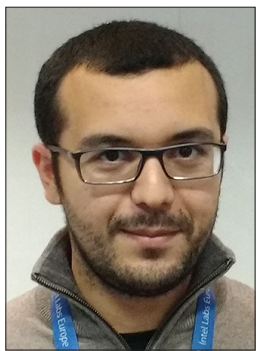

Wael Guibene

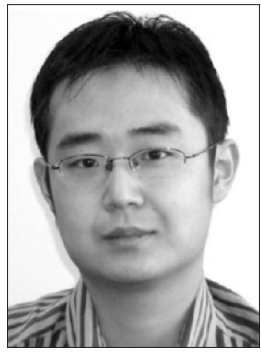

Tom H. Luan

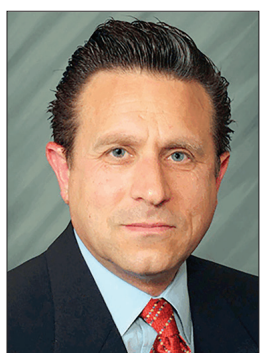

Ammar Rayes ue to the random vehicle mobility and varying communication environment, an integrated vehicular network comprising heterogeneous access technologies (DSRC, WiFi, 4G/LTE, 5G, etc.) will be indispensable to provide ubiquitous mobile coverage. Although heterogeneous networking has been extensively studied in different contexts, the salient features of vehicular communications (e.g., varying road density, fast mobility) have brought new challenges and led to fundamental and interesting research issues, such as how to flexibly configure and efficiently conduct resource allocation, how to enable interoperation among multiple coexisting networks, and how to effectively accommodate a large number of traveling users with various kinds of smart devices.

In addition to the advances in the underlying access technologies, cloud computing as a centralized control and management solution has become mature, representing an indispensable component of large scale vehicular networks. In particular, software-defined networking (SDN) has emerged as a promising paradigm to control the network in a systematic way, gaining attention from both academia and industry. The flexibility and programmability of SDN not only make it attractive to satisfy the quality of service (QoS) requirements of vehicular services, but also greatly simplify resource management in heterogeneous vehicular networks.

In this Feature Topic (FT), we invited authors from the industry and academic research communities to discuss the architecture, applications, challenges, and standardization efforts on enabling software-defined vehicular networking (SDVN). We received a large number of submissions and conducted a rigorous review process. This is Part 2 of the FT.
To deliver content to remote vehicles, path establishment, maintenance, and identity assignment in dynamic vehicular networks generate much overhead. S. H. Ahmed et al., in "Named Data Networking for Software Defined Vehicular Networks," present an architecture that utilizes SDN in vehicular networks to support content retrieval by named data networking. Furthermore, a number of current research challenges are discussed, and a precise roadmap to address these issues is provided.

The high user mobility in vehicular networks usually results in long handover delay among eNodeBs (eNBs) in 5G communications. C. F. Lai et al., in "A Buffer-Aware QoS Streaming Approach for SDN-Enabled 5G Vehicular Networks," propose a buffer-aware QoS streaming approach over SDN-enabled 5G vehicular networks, which provides various priority levels of streaming services to mobile users. Experimental results show that the proposed approach can adjust the priority of streaming content segments and avoid the delay of streaming content transmissions for $5 \mathrm{G}$ vehicular networks.

In order to support software updates in vehicles, M. Azizian et al. propose an SDN-based vehicular cloud architecture (SVC) that leverages vehicle-to-vehicle (V2V) communications in "Vehicle Software Updates Distribution with SDN and Cloud Computing." In SVC, solutions on how vehicular networks can be modeled as connectivity graphs are proposed, and an SDN-based scheme that assigns different frequency bands to graph edges is presented to improve network performance.

The highly coupled design of traditional networks cannot satisfy various QoS requirements in vehicular networks. W. 
Quan et al., in "Enhancing Crowd Collaborations for Software Defined Vehicular Networks," propose a customized smart identifier networking (SINET)-based solution to enable crowd collaboration for SDVN. It utilizes crowd sensing to flexibly select virtualized function slices, and enables crowd collaboration to dynamically adapt to various vehicular scenarios and applications.

In edge networks, a network may suffer from potential interference, resource congestion, or underutilization in the absence of joint resource optimization. D. J. Deng et al., in "Latency Control in Software Defined Mobile Edge Vehicular Networking," point out a complete series of latency control mechanisms and principles for edge-up software-defined cloud/edge vehicular networking. The proposal thus creates a paradigm shift to enable SDVN.

Drivers' fatigue and their mood shifts may lead to traffic accidents. Y. Zhang et al., in "SOVCAN: Safety-Oriented Vehicular Controller Area Network," propose an SDNbased approach to develop a safety-oriented vehicular controller area network (SOVCAN), which can improve traffic safety by detecting drivers' fatigue and recognize their emotions.

In order to offload the data to centralized servers or other devices, an intelligent controller is indispensable to make decisions. G. S. Aujla et al., in "Data Offloading in 5G-Enabled Software-Defined Vehicular Networks: A Stackelberg-Game-Based Approach," propose a novel data offloading scheme for 5G-enabled SDN-based vehicular networks. The SDN-based controller makes decisions for data offloading by using the priority manager and load balancer. In addition, a single leader multi-follower Stackelberg game is designed for network selection. The performance evaluations of the proposed scheme demonstrate the superiority of the scheme to other existing proposals.

In closing, we would like to thank all of those who have made significant contributions to this $\mathrm{FT}$, including the contributing authors, the anonymous reviewers, and the IEEE Communications Magazine publications staff, in particular the Editor-in-Chief. We believe that the research results presented in this FT will further stimulate research and development ideas in vehicular networks.

\section{BIOGRAPHIES}

GUANGIIE HAN [S'01, $\mathrm{M}^{\prime} 05$ ] is currently a professor with the Department of Information and Communication Systems, Hohai University, China. His current research interests include sensor networks, computer communications, mobile cloud computing, and multimedia communication and security. He has served on the Editorial Boards of up to 14 international journals, including IEEE Access and Telecommunication Systems. He has been a Guest Editor for a number of Special Issues in IEEE journals and magazines. He is a member of ACM

MOHSEN GUIZANI [S'85, M'89, SM'99, F'09] received his B.S, M.S., and Ph.D. from Syracuse University. He is currently a professor and the ECE Department Chair at the University of Idaho. His research interests include wireless communications/ mobile cloud computing, computer networks, security, and smart grid. He is the author of nine books and more 450 publications. He was Chair of the IEEE Communications Society Wireless Technical Committee. He served as an IEEE Computer Society Distinguished Speaker.

YuANGUO Bı received his Ph.D. degree from Northeastern University, Shenyang, China, in 2010. He joined the School of Computer Science and Engineering, Northeastern University, as an associate professor in 2010. His current research interests focus on medium access control, QoS routing, multihop broadcast, mobility management in vehicular networks, as well as SDN-enabled vehicular networks.

TOM H. LUAN received his Ph.D. degree from the University of Waterloo, Ontario, Canada, in 2012. Since December 2013, he has been a lecturer in mobile and apps with the School of Information Technology, Deakin University, Melbourne, Australia. His research mainly focuses on vehicular networking, wireless content distribution, peer-to peer networking, and mobile cloud computing.

KAORU OTA received Ph.D. degrees in computer science and engineering from the University of Aizu, Japan, in 2012. She is currently an assistant professor with the Department of Information and Electronic Engineering, Muroran Institute of Technology, Japan. She was a research scientist with the A3 Foresight Program (2011-2016) funded by the Japan Society for the Promotion of Sci-ences (JSPS), NSFC of China, and NRF of Korea.

HAIBO ZHOU received his Ph.D. degree in information and communication engineering from Shanghai Jiao Tong University, China, in 2014. From 2014 to 2016 he was a postdoctoral research fellow with the Broadband Communications Research (BBCR) Group, University of Waterloo. He is currently a research associate in the BBCR Group. His current research interests include resource management and protocol design in cognitive radio networks and vehicular networks.

AMMAR RAYES [S'85, M'91, SM'15] is a Distinguished Engineer focusing on the technology strategy for Cisco Services. His research interests include loT, network management NMS/OSS, machine learning, analytics, and security. He has authored three books, over 100 publications in refereed journals and conferences on advances in software and networking related technologies, and over 25 patents. He received B.S. and M.S. degrees from the University of Illinois at Urbana and D.Sc. degree from Washington University, all in electrical engineering.

WAEL GUIBENE is a research scientist at Intel Labs since June 2015. He was awarded his Ph.D. from Telecom ParisTech in July 2013. He also holds an M.Eng. and a Master's degree in telecommunications obtained in 2009 and 2010, respectively. He worked at Eurecom as a research engineer from 2010 to November 2013 and then joined Semtech to work on LoRa systems from 2013 to June 2015. His research activities include $\mathrm{loT}, 5 \mathrm{G}$, and wireless communications. 